Pacific Journal of Mathematics

SPECTRAL THEORY FOR A FIRST-ORDER SYMMETRIC
SYSTEM OF ORDINARY DIFFERENTIAL OPERATORS 


\title{
SPECTRAL THEORY FOR A FIRST-ORDER SYMMETRIC SYSTEM OF ORDINARY DIFFERENTIAL OPERATORS
}

\author{
SORRELL BERMAN
}

For a symmetric differential expression associated with a first order system

$$
A_{0}(t) x^{\prime}+A(t) x, a<t<b
$$

where $A_{0}$ and $A$ are $n \times n$ matrices and $x$ is an $n \times 1$ vector, a spectral decomposition will be developed. That is, if $S$ is a closed symmetric differential operator determined by the differential system, the explicit nature of the generalized resolutions of the identity for all the self-adjoint extensions of $S$ in any Hilbert space will be determined in terms of a fundamental matrix and spectral matrices associated with these extensions. An important aspect is that these self-adjoint extensions may be defined in Hilbert spaces larger than the natural one $\mathscr{H}$ in which the operator $S$ is defined.

The development proceeds as in Coddington [5]; however, the consideration of systems of differential equations introduces matrix techniques and notation. It is hoped that this formulation will have application to such problems as open end (infinite time) control theory problems, and facilitate the canonical formulation of the associated spectral analysis.

Preliminary definitions. Let $\mathscr{H}$ be a Hilbert space with an inner product (, ).

(1) Generalized Resolution of the Identity. Let $F=\{F(\lambda)\}$ be a family of bounded self-adjoint operators in $\mathscr{H}$, depending on real $\lambda$, such that:

(i) $F(\lambda) \geqq F(\mu), \lambda>\mu$,

(ii) $F(\lambda+0)=F(\lambda)$,

(iii) $F(\lambda) \rightarrow I$, as $\lambda \rightarrow+\infty$, $F(\lambda) \rightarrow 0$, as $\lambda \rightarrow-\infty$,

then $F$ is a generalized resolution of the identity.

The family $F$ is said to be associated with a symmetric operator $Z$ (or $F$ is a "spectral function" for $Z$, Naimark [7]) if

$$
(Z u, v)=\int \lambda d(F(\lambda) u, v),
$$




$$
\|Z u\|^{2}=\int \lambda^{2} d(F(\lambda) u, u),
$$

for all $u \in \mathscr{D}(Z)$ and $v \in \mathscr{H}$.

(2) Generalized Resolvent. Let $Z$ be a symmetric operator and $F=\{F(\lambda)\}$ be an associated generalized resolution of the identity. For $\operatorname{Im} l \neq 0$, let $\mathscr{R}=\{\mathscr{R}(l)\}$ be a family of operators such that

$$
(\mathscr{R}(l) u, v)=\int \frac{d(F(\lambda) u, v)}{\lambda-l} .
$$

Then $\mathscr{R}$ is a generalized resolvent of $Z$ associated with $F$. The development for symmetric operators will include the case for selfadjoint operators.

1. Basic vector and matrix definitions. In addition to the usual definitions and notation for the absolute magnitude of a vector, the inner product of two vectors, the norm of a vector and the absolute magnitude of a matrix, the norm of a matrix is defined as

$$
\|A\|=\left(\left.\left.\sum_{i=1}^{n} \sum_{j=1}^{n} \int\right|_{1} A_{i j}(t)\right|^{2} d t\right)^{1 / 2}=\left(\int \operatorname{trace}\left(A^{*}(t) A(t)\right) d t\right)^{1 / 2} ;
$$

and a matrix "inner product" is introduced,

$$
(A, B)=\int B^{*}(t) A(t) d t
$$

which is a matrix whose $(i, j)$ th element is

$$
\sum_{l=1}^{n} \int \bar{B}_{l i}(t) A_{l j}(t) d t
$$

This "inner product" makes sense for any two matrices for which $B^{*}(t) A(t)$ exists and is integrable.

An inner product of a matrix and a vector can be defined in some situations; it is a special case of the matrix "inner product." For example, if $f$ is an $n \times 1$ vector and $G$ an $n \times n$ matrix,

$$
(f, G)=\int G^{*}(t) f(t) d t
$$

2. The "basic operators" $T_{0}$ and $T$. Let $(a, b)$ be an open interval on the real line ( $a$ may be $-\infty$ and/or $b$ may be $+\infty$ ). A differential operator $L$ is defined by

$$
L x=A_{0}(t) x^{\prime}+A(t) x .
$$

where: $x$ is an $n \times 1$ vector, $A_{0}$ and $A$ are $n$ by $n$ suitably regular 
matrix-valued functions (Brauer [2]) and ' denotes $d / d t$. The Lagrange adjoint, $L^{+}$, associated with $L$ is defined by

$$
\begin{aligned}
L^{+} y & =-\left(A_{0}^{*}(t) y\right)^{\prime}+\left(A^{*}(t) y\right) \\
& =-A_{0}^{*}(t) y^{\prime}+\left(-A_{0}^{* \prime}(t)+A^{*}(t) y\right) .
\end{aligned}
$$

The operator $L$ is formally self-adjoint if $L=L^{+}$, that is when

$$
A_{0}=-A_{0}^{*} \text { and } A=-A_{0}^{* \prime}+A^{*}=A_{0}^{\prime}+A^{*} .
$$

Throughout the remainder of this paper $L$ will be assumed to be formally self-adjoint.

Using the definitions for the inner product of two vectors, and for the norm, a Hilbert space, $\mathscr{H}$, can be defined,

$$
\mathscr{H}=\mathscr{L}^{2}(a, b)=\{u:\|u\|<\infty\} .
$$

Defining a domain $\mathscr{D}$ in $\mathscr{H}$ by $\mathscr{D}=\{u \in \mathscr{H}:$ ( i ) $u$ is absolutely continuous on every compact subinterval of $(a, b)$, (ii) $L u \in \mathscr{H}$ \}, an operator $T$, having domain $\mathscr{D}$, can be defined by

$$
T u=L u, \quad u \in \mathscr{D} .
$$

Let, for $u, v \in \mathscr{D}$,

$$
\langle u v\rangle=(L u, v)-\left(u, L^{+} v\right)=(L u, v)-(u, L v) .
$$

Then, similarly for a domain $\mathscr{D}_{0}$,

$$
\mathscr{D}_{0}=\{u \in \mathscr{D}:\langle u v\rangle=0 \text { for all } v \in \mathscr{D}\},
$$

an operator $T_{0}$ can be defined by

$$
T_{0} u=L u, \quad u \in \mathscr{D}_{0} .
$$

The development of the operators $T_{0}$ and $T$ is motivated by the fact that $T_{0}$ is the smallest closed symmetric operator in $\mathscr{H}$ (associated with the differential operator $L$ ) having a domain which contains all vectors which are infinitely differentiable on $(a, b)$ and vanish outside closed bounded subintervals of $(a, b)$. Further, if $F_{1}$ is any generalized resolution of the identity for a closed symmetric operator $T_{1}$, where $T_{0} \subset T_{1} \subset T$, then $F_{1}$ is a generalized resolution of the identity for $T_{0}$, also. Thus, by considering $T_{0}$, a maximal set of generalized resolutions of the identity, which are naturally associated with $L$, can be obtained. The following theorem provides an important relation between $T_{0}$ and $T$.

THEOREM 2.1. The operator $T_{0}$ is closed, symmetric, and $T_{0}^{*}=$ 
$T, T^{*}=T_{0}$

Proof. Let

$$
K(t, \tau)= \begin{cases}\Phi(t) \Phi^{-1}(\tau) A_{0}^{-1}(\tau), & t \geqq \tau, \\ 0, & t<\tau,\end{cases}
$$

where $\Phi$ is a fundamental matrix, that is, a matrix whose columns are independent solutions of $L x=0$. Thus, $\Phi$ is a nonsingular $n \times n$ matrix such that $L \Phi=0$. As a function of $t, L K(t, \tau)=0$, and

$$
K(t+, t)-K(t-, t)=\Phi(t+) \Phi^{-1}(t) A_{1}^{-0}(t)-0=A_{0}^{-1}(t) .
$$

The representation for $K$ can be simplified

$$
\left(\Phi^{*} A_{0} \Phi\right)^{\prime}=0 \text {, or } \Phi^{*} A_{0} \Phi=D^{-1},
$$

where $D$ is a skew-Hermitian constant matrix, and hence

$$
\Phi^{-1} A_{0}^{-1}=D \Phi^{*} .
$$

The matrix $K$ can now be written as

$$
K(t, \tau)= \begin{cases}\Phi(t) D \Phi^{*}(\tau), & t \geqq \tau \\ 0, & t<\tau .\end{cases}
$$

Let $\Delta$ be a closed bounded subinterval $[\widetilde{a}, \widetilde{b}]$ of $(a, b)$. The Hilbert space $\mathscr{L}^{2}(\Delta)$ is defined by

$$
\mathscr{L}^{2}(\Delta)=\left\{u:\|u\|_{\Delta}<\infty\right\} .
$$

For $t \in \Delta$, the vector $x$ defined by

$$
\begin{aligned}
x(t) & =\int_{\widetilde{a}}^{\widetilde{b}} K(t, \tau) y(\tau) d \tau \\
& =\int_{\widetilde{a}}^{t} K(t, \tau) y(\tau) d \tau,
\end{aligned}
$$

where $y \in \mathscr{L}^{2}(\Delta)$, is such that

(0) $x \in \mathscr{L}^{2}(\Delta)$,

(i) $x$ is absolutely continuous on $\Delta$,

(ii) $L x \in \mathscr{L}^{2}(\Delta)$.

Having verified that for $t \in \Delta$ and $y \in \mathscr{L}^{2}(\Delta)$ the vector $x$ satisfies conditions ( 0 ), ( $\mathrm{i})$, and ( $\left.\mathrm{ii}_{i}^{i}\right)$, the proof follows exactly as in Theorem 1 of reference 3 .

3. The Green's function $G_{\Delta^{*}}$. In $\S 5$. the generalized resolvents associated with $T_{0}$ will be constructed. The generalized resolvent will be developed starting from the Green's function $G_{\Delta}$ associated with certain self-adjoint boundary-value problems on finite subintervals 
4. The purpose of this section is to derive such Green's functions $G_{\Delta}$.

Once again, let $\Delta$ be a closed bounded subinterval of $(a, b)$, denoted by $[\widetilde{a}, \tilde{b}]$. Analogous to previous definitions, a domain $\mathscr{D}_{\Delta}$ is defined and the associated operator $T_{\Delta}$, having domain $\mathscr{D}_{\Delta}$,

$$
T_{\Delta} u=L u, \quad u \in \mathscr{D}_{\Delta} .
$$

Similarly, for $\mathscr{D}_{04}$, an operator $T_{04}$, having domain $\mathscr{D}_{04}$, is defined by

$$
T_{04} u=L u, \quad u \in \mathscr{D}_{04} .
$$

[NOTE: The conditions and relations of Theorem 2.1 hold for $T_{04}$ and $\left.T_{\Delta} \cdot\right]$

It will now be shown that abstract self-adjoint boundary conditions can be constructed by considering the self-adjoint extensions of $T_{04}$. Let

$$
\mathscr{E}_{\Delta}( \pm i)=\left\{v \in \mathscr{D}_{\Delta}: T_{\Delta} v= \pm i v\right\}
$$

It is clear that $\operatorname{dim} \mathscr{E}_{\Delta}(i)=\operatorname{dim} \mathscr{E}_{\Delta}(-i)=n$. The domain $\mathscr{D}_{\Delta}$ can be written as a direct sum

$$
\mathscr{D}_{\Delta}=\mathscr{D}_{04}+\mathscr{E}_{4}(i)+\mathscr{E}_{\Delta}(-i) .
$$

From the theory of the Cayley transform (see Riesz-Nagy [8], for example) every self-adjoint extension, $T_{\Delta U}$, of $T_{0 \Delta}$ has a domain

$$
\mathscr{D}_{\Delta U}=\mathscr{D}_{04}+(I-U) \mathscr{E}_{\Delta}(-i),
$$

where $U$ is a unitary mapping from $\mathscr{E}_{4}(-i)$ onto $\mathscr{E}_{\Delta}(i)$; and

$$
T_{v U} u=L u, \quad u \in \mathscr{E}_{\Delta U} .
$$

Let $\left\{\varphi_{\Delta i}\right\} i=1, \cdots, n$, be an orthonormal basis for $\mathscr{E}_{\Delta}(i)$; also let $\left\{\psi_{\Delta i}\right\} i=1, \cdots, n$, be an orthonormal basis for $\mathscr{E}_{\Delta}(-i)$; finally let

$$
v_{\Delta j}=\psi_{\Delta j}-U \psi_{\Delta j},
$$

and

$$
v_{\Delta j^{*}}=\varphi_{\Delta j}-U^{*} \phi_{\Delta j}, \quad j=1, \cdots, n .
$$

The following theorem describes the abstract self-adjoint boundary conditions induced by the domain $\mathscr{D}_{\Delta U}$.

THEOREM 3.1. The domain $\mathscr{D}_{\Delta U}$ of $T_{\Delta U}$ has the following representation:

$$
\mathscr{D}_{\Delta U}=\left\{u \in \mathscr{D}:\left\langle u v_{\Delta j *}\right\rangle=0, \mathrm{j}=1, \cdots, n\right\}
$$

where $\left\{\left\langle u v_{\Delta j}\right\rangle=0, j=1, \cdots, n\right\}$ form a self-adjoint set of boundary 
conditions.

Proof. This follows by direct analogy from the proof of Theorem 3 in Coddington [3].

The set $\left\langle\left\langle u v_{\Delta j} *\right\rangle=0, j=1, \cdots, n\right\}$ forms a self-adjoint set of boundary conditions since the $v_{\Delta j} *$ are linearly independent and $\left\langle v_{\Delta j} * v_{\Delta k} *\right\rangle$ $=0$ for all $j, k$.

The set of self-adjoint boundary conditions $\left\langle\left\langle u v_{\Delta j}\right\rangle=0, j=1, \cdots\right.$, $n$ ) can be represented in matrix form by

$$
V_{\Delta *}^{*}(\widetilde{b}) A_{0}(\tilde{b}) u(\tilde{b})-V^{*}(\widetilde{a}) A_{0}(\widetilde{a}) u(\widetilde{a})=0,
$$

where $V_{\Delta *}$ is the matrix whose $i$ th column is the vector $v_{\Delta i} *$. Letting

$$
M_{\Delta}=-V_{\Delta *}^{*}(\widetilde{a}) A_{0}(\widetilde{a}),
$$

and

$$
N_{\Delta}=V_{\Delta *}^{*}(\widetilde{b}) A_{0}(\tilde{b})
$$

the self-adjoint boundary conditions can be written in standard form

$$
U_{\Delta} u=M_{\Delta} u(\widetilde{a})+N_{\Delta} u(\widetilde{b})=0 .
$$

The self-adjoint boundary-value problem (on $\Delta$ )

$$
L u=l u, \quad U_{\Delta} u=0 \quad(b v)
$$

will now be considered. The Green's function $G_{\Delta}$ associated with the problem $(b v)$ is a unique function $G_{\Delta}(t, \tau, l)$ ( $l$ not an eigenvalue of $(b v)$ ) satisfying the following conditions:

(i) $G_{\Delta}(t, \tau, l)$ and $\partial / \partial t G_{\Delta}(t, \tau, l)$ are continuous on $\widetilde{a} \leqq t \leqq \tau \leqq \tilde{b}$ and $\tilde{a} \leqq \tau \leqq t \leqq b$, and for each fixed $(t, \tau)$ are analytic in $l$,

(ii) $G_{\Delta}(t+, \tau, l)-G_{\Delta}(t-, t, l)=A_{0}^{-1}(t), \widetilde{a}<t<\widetilde{b}$,

(iii) $G_{\Delta}$ satisfies $L G_{\Delta}=l G_{\Delta}$ (as a function of $t$ ),

(iv) $G_{\Delta}$ satisfies $U_{\Delta} G_{\Delta}=0$ (as a function of $t$ ),

(v) $G_{\Delta}(t, \tau, l)=G_{\Delta}^{*}(\tau, t, \bar{l})$,

(vi) if $f \in \mathscr{L}^{2}(\Delta)$ and $L u=l u+f$, then,

$$
u(t)=\int_{\Delta} G_{\Delta}(t, \tau, l) f(\tau) d \tau, \quad U_{\Delta} u=0
$$

and if

$$
\mathscr{G}_{\Delta}(l) f(t)=\int_{\Delta} G_{\Delta}(t, \tau, l) f(\tau) d \tau,
$$

then 


$$
(L-l) \mathscr{G}_{\Delta}(l) f(t)=f(t),
$$

and

$$
\left\|\mathscr{G}_{\Delta}(l)\right\|_{\Delta} \leqq|\operatorname{Im} l|^{-1}
$$

The Green's function will now be constructed starting from the kernel

$$
K_{\Delta}(t, \tau, l)= \begin{cases}\Phi(t, l) D \Phi^{*}(\tau, l), & t \geqq \tau \\ 0, & t<\tau,\end{cases}
$$

for $t, \tau \in \Delta$; where $\Phi$ is a fundamental matrix for $(L-l) u=0$, having the property that for some $c, \tilde{a}<c<\tilde{b}, \Phi(c, l)=I$. The matrix $D(=$ $\left.A_{0}^{-1}(c)\right)$ is a constant, skew-Hermitian matrix. From Theorem 8.4 Coddington and Levinson [6], $\Phi$ is continuous as a function of $(t, l)$, and for fixed $t$ is an analytic function of $l(\operatorname{Im} l \neq 0)$. Let

$$
G_{\Delta}(t, \tau, l)=K_{\Delta}(t, \tau, l)+\Phi(t, l) J(\tau, l) .
$$

Introducing the notation

$$
U_{\Delta} \Phi(l)=M_{\Delta} \Phi(\widetilde{a}, l)+N_{\Delta} \Phi(\tilde{b}, l),
$$

$G_{\Delta}$ can be written as

$$
G_{\Delta}(t, \tau, l)=\left\{\begin{array}{l}
\Phi(t, l)\left(U_{\Delta} \Phi(l)\right)^{-1} M_{\Delta} \Phi(\widetilde{a}, l) D \Phi^{*}(\tau, \bar{l}), \quad t \geqq \tau \\
-\Phi(t, l)\left(U_{\Delta} \Phi(l)\right)^{-1} N_{\Delta} \Phi(\widetilde{b}, l) D \Phi^{*}(\tau, \bar{l}), \quad t<\tau .
\end{array}\right.
$$

It now follows by direct verification that $G_{4}$ as constructed satisfies the remaining five conditions.

4. The limit function $G$. In this section it will be shown that a type of limit function $G$ exists for the set $\left\{G_{\Delta}\right\}$, as $\Delta$ approaches $(a, b)$.

Let $\Delta_{0}, \Delta_{1}$, and $\Delta$ be closed bounded subintervals of $(a, b)$ such that $\Delta_{0}$ is properly contained in $\Delta_{1}$, and $\Delta_{1}$ is properly contained in $\Delta$; these will be denoted by

$$
\Delta_{0}=\left[a_{0}, b_{0}\right], \Delta_{1}=\left[a_{1}, b_{1}\right], \Delta=[\tilde{a}, \tilde{b}] .
$$

Let $\mu$ be a function, having a continuous first derivative, such that for some open interval $\Delta_{2}, \Delta_{0} \subset \Delta_{2} \subset \Delta_{1}$

$$
\mu(t)= \begin{cases}1, & t \in \Delta_{2} \\ 0, & t \text { outside } \Delta_{1} .\end{cases}
$$

Let 


$$
W_{\Delta}(t, \tau, l)=G_{\Delta}(t, \tau, l)-\mu(t) G_{\Delta_{1}}(t, \tau, l) .
$$

Then for $t, \tau \in \Delta_{0}, W_{\Delta}(t, \tau, l)$ is continuous; as a function of $t, W_{\Delta}$ satisfies

$$
U_{\Delta} W_{\Delta}=M_{\Delta} W_{\Delta}(\widetilde{a})+N_{\Delta} W(\widetilde{b})=0 ;
$$

and also

$$
\left(L_{t}-l\right) W_{\Delta}(t, \tau, l)=-A_{0}(t) \mu^{\prime}(t) G_{\Delta_{1}}(t, \tau, l), \quad t \neq \tau .
$$

Since $\mu^{\prime}(t)=0$ for $t$ outside of $\Delta_{1}, W_{\Delta}$ can be written as

$$
W_{\Delta}(t, \tau, l)=-\int_{\Delta_{1}} G_{\Delta}(t, s, l) A_{0}(s) \mu^{\prime}(s) G_{\Delta_{1}}(s, \tau, l) d s ;
$$

(Note: The integral over $\Delta_{1}$ actually represents the sum of integrals over $[a, t-],[t+, \tau-],[\tau+, b]$ for $\tau>t)$, or

$$
G_{\Delta}(t, \tau, l)=\mu(t) G_{\Delta_{1}}(t, \tau, l)-\int_{\Delta_{1}} G_{\Delta}(t, s, l) A_{0}(s) \mu^{\prime}(s) G_{\Delta_{1}}(s, \tau, l) d s .
$$

It can be shown that the set $\left\{W_{\Delta}\right\}$ is uniformly bounded and equicontinuous on any compact $(t, \tau, l)$ - region, $\operatorname{Im} l \neq 0, t \neq \tau$. Thus, by Ascolis' theorem a uniform limit $W$ exists and from this a limit function $G$, where

$$
G=\mu G_{\Delta_{1}}+W
$$

and $G$ is a limit function for the set $\left\{G_{A}\right\}$.

THEOREM 4.1. The function $G$ satisfies the following conditions:

(i) $G(t, \tau, l)$ and $\partial / \partial t G(t, \tau, l)$ are continuous on $a<t \leqq \tau<b$ and $a<\tau \leqq t<b$, and for $\operatorname{Im} l \neq 0 \quad G$ is analytic in $l$,

(ii) $G(t+, t, l)-G(t-, t, l)=A_{0}^{-1}(t), a<t<b$,

(iii) $L_{t} G=l G, t \neq \tau$,

(iv) $G(t, \tau, l)=G^{*}(\tau, t, \bar{l})$,

(v) $G(t,, l) \in \mathscr{L}^{2}(a, b), a<t<b$,

(vi) If $f \in \mathscr{L}^{2}(a, b)$, then the vector $v$ defined by

$$
v(t)=\int_{a}^{b} G(t, \tau, l) f(\tau) d \tau, \quad \operatorname{Im} l \neq 0,
$$

is such that $v \in \mathscr{D}$ and

$$
L v(t)=l v(t)+f(t) .
$$

Proof. Again, this follows by direct verification.

It is thus seen that $G$ satisfies 'all the conditions of a Green's 
function except for satisfying boundary conditions. Further, from property (vi), if

$$
\mathscr{G}(l) f(t)=\int_{a}^{b} G(t, \tau, l) f(\tau) d \tau,
$$

then,

$$
(L-l) \mathscr{G}(l) f(t)=f(t)
$$

and $\mathscr{G}(l)$ is a right inverse for $L-l$.

5. The generalized resolvent. Having constructed the closed symmetric operator $T_{0}$, all its self-adjoint extensions will now be considered. In §3. the self-adjoint extensions for an operator in $\mathscr{H}$ having equal deficiency indices were considered and these self-adjoint extensions were also in the space $\mathscr{H}$. A spectral analysis of those self-adjoint extensions occurring in $\mathscr{H}$ was carried out, by quite different methods, by Brauer in [2]. The problem to be considered next is for unequal deficiency indices or equivalently, singular problems with equal deficiency indices such that the self-adjoint extensions are outside the original space.

Naimark [7] and others have defined extensions of $T_{0}$ for this case in larger Hilbert spaces. Theorem 7. in Straus [12] provides a means for an explicit construction in $\mathscr{C}$ itself. Let $A(l)$ map $\mathscr{E}(-i)$ into $\mathscr{E}(i)$, where $A(l)$ is analytic and $\|A(l)\| \leqq 1$ for $\operatorname{Im} l>0$. Analogously to the case of equal deficiency indices, a domain $\mathscr{D}(l) \subset \mathscr{D}$ is defined by

$$
\mathscr{D}(l)=\mathscr{D}_{0}+(I-A(l)) \mathscr{E}(-i),
$$

and an operator $T_{A(l)}$, having domain $\mathscr{D}(l)$ is defined by

$$
T_{A(l)} u=T u, \quad u \in \mathscr{D}(l) .
$$

Then, $T_{0} \subset T_{A(l)} \subset T$, and the generalized resolvent $\mathscr{R}$ can be represented as

$$
\mathscr{R}(l)=\left(T_{A(l)}-l I\right)^{-1}, \mathscr{R}(\bar{l})=\mathscr{R}^{*}(l), \operatorname{Im} l>0 .
$$

Further, from Straus [12] every generalized resolvent is generated by such $A(l)$.

Again, analogously to the case for equal deficiency indices, the domain $\mathscr{D}(l)$ can be characterized in an alternate manner which leads to an explicit formulation for the generalized resolvent $\mathscr{R}(l)$. The domain $\mathscr{D}$ can be represented as a direct sum

$$
\mathscr{D}=\mathscr{D}_{0}+\mathscr{E}(i)+\mathscr{E}(-i)
$$


let $\omega^{+}$be the dimension of $\mathscr{E}(i)$, and $\omega^{-}$be the dimension of $\mathscr{E}(-i)$, where $0 \leqq \omega^{+}, \omega^{-} \leqq n$; let $\left\{\varphi_{j}(i)\right\}, j=1, \cdots, \omega^{+}$, be an orthonormal basis for $\mathscr{E}(i)$, and let $\left\{\psi_{k}(-i)\right\}, k=1, \cdots, \omega^{-}$, be an orthonormal basis for $\mathscr{E}(-i)$; finally, let

$$
v_{j}(l)=\psi_{j}(-i)-A(l) \psi_{j}(-i),
$$

and

$$
v_{j}(l)=\varphi_{j}(i)=A^{*}(l) \varphi_{j}(i) .
$$

TheOREM 5.1. For $\operatorname{Im} l>0$, the domain $\mathscr{D}(l)$ of $T_{A(l)}$ can be represented as

$$
\mathscr{D}(l)=\left\{u \in \mathscr{D}:\left\langle u v_{j *}(l)\right\rangle=0, \quad j=1, \cdots, \omega^{+}\right\} ;
$$

and the domain of $T_{A(l)}^{*}$ is

$$
\mathscr{D}^{*}(l)=\left\{w \in \mathscr{D}:\left\langle w v_{k}(l)\right\rangle=0, \quad k=1, \cdots, \omega^{-}\right\} .
$$

Proof. The proof is the same as the proof of Theorem 3.1 with the operator $A(l)$ in place of $U$.

It will now be shown, again analogous to $\S 3$, that the domain $\mathscr{D}(l)$ induces limiting abstract boundary conditions. For $u \in \mathscr{D}$ and any closed bounded subinterval $[c, d]$ of $(a, b)$

$$
\left[u v_{j *}\right](d)-\left[u v_{j *}\right](c)=v_{j *}^{*}(d) A_{0}(d) u(d)-v_{j *}^{*}(c) A_{0}(c) u(c) .
$$

Since $u, v_{j *}, L u$, and $L v_{j *}$ are each in $\mathscr{L}^{2}(a, b)$, then $\lim _{d \rightarrow b} v_{j *}^{*}(d) A_{0}(d) u(d)$ exists, and $\lim _{c \rightarrow a} v_{j *}^{*}(c) A_{0}(c) u(c)$ exists; these limits will be denoted by $v_{j *}^{*}(b) A_{0}(b) u(b)$ and $v_{j *}^{*}(a) A_{0}(a) u(a)$. The conditions $\left\langle\left\langle u v_{j *}\right\rangle=0, j=\right.$ $\left.1, \cdots, \omega^{+}\right\}$can then be represented in matrix form as

$$
0=\left\langle u V_{*}\right\rangle=V_{*}^{*}(b, l) A_{0}(b) u(b)-V_{*}^{*}(a, l) A_{0}(a) u(a)(L B),
$$

where $V_{*}$ is the matrix with $v_{j *}$ in the $j$ th column; these are limiting abstract boundary conditions.

Having obtained the limiting abstract boundary conditions, the following theorem describes a method for the construction of the generalized resolvent $\mathscr{R}(l)$ starting from the integral operator $\mathscr{G}(l)$ developed in $\S 4$.

THEOREM 5.2. Each generalized resolvent $\mathscr{R}(l)$ of $T_{0}$ is an integral operator of Carleman type, having a kernel $R(t, \tau, l)$, which is continuous in $(t, \tau, l)$ and analytic in $l$ in any region for which $\operatorname{Im} l \neq 0$, and $t \neq \tau$.

Proof. The integral operator $\mathscr{G}(l)$ obtained in $\S 4$. is of Carleman 
type; further $\mathscr{G}(l)$ satisfies the conditions of the theorem except that $\mathscr{G}(l)$ is only a right inverse of $(T-l)$. It will now be shown that a matrix $G_{1}$ can be constructed such that the kernel of $\mathscr{R}(l)$ is

$$
R(t, \tau, l)=G(t, \tau, l)+G_{1}(t, \tau, l) .
$$

For fixed $l, \operatorname{Im} l>0$, let $\left\{\theta_{i}(l)\right\}, i=1, \cdots, \omega^{+}$, be an orthonormal basis for $\mathscr{E}(l)$, let $\Theta_{+}(l)$ be the $n \times \omega^{+}$matrix having $\theta_{j}(l)$ in the $j$ th column, similarly, let $\left\{\chi_{k}(\bar{l})\right\}, k=1, \cdots, \omega^{-}$, be an orthonormal basis for $\mathscr{E}(\bar{l})$, and $\chi_{-}(\bar{l})$ be the $n \times \omega^{-}$matrix having $\chi_{k}(\bar{l})$ in the $k$ th column. From the orthonormal property of the $\theta_{i}(l)$ and the $\chi_{k}(\bar{l})$,

$$
\begin{aligned}
\left(\Theta_{+}(l), \Theta_{+}(l)\right) & =\int_{a}^{b} \Theta_{+}^{*}(t, l) \Theta_{+}(t, l) d t \\
& =I_{\omega^{+} \times \omega^{+}}
\end{aligned}
$$

where $I_{\omega^{+} \times \omega^{+}}$is the identity matrix of rank $\omega^{+}$; similarly,

$$
\left(\chi_{-}(\bar{l}), \chi_{-}(\bar{l})\right)=I_{\omega^{-\times \omega^{-}}} .
$$

For any vector $f$ in $\left.\mathscr{L}^{2}(a, b),(T-l)(\mathscr{R}(l)-\mathscr{G}(l)) f\right)=f-f=$ 0 , and thus $(\mathscr{R}(l)-\mathscr{G}(l)) f$ is in $\mathscr{E}(l)$. Thus for some $\omega^{+} \times 1$ vector $a(f, l)$

$$
(\mathscr{R}(l)-\mathscr{G}(l)) f=\Theta_{+}(l) a(f, l) .
$$

Also

$$
\left(\left(\mathscr{R}(l)-\mathscr{G}(l) f, \Theta_{+}(l)\right)=\left(f,(\mathscr{R}(\bar{l})-\mathscr{G}(\bar{l})) \Theta_{+}(l)\right) ;\right.
$$

and, for each column $\theta_{k}(l)$ of $\Phi_{+}(l),(T-\bar{l})(\mathscr{R}(\bar{l})-\mathscr{G}(\bar{l})) \theta_{k}(l)=\theta_{k}(l)$ $-\theta_{l}(l)=0$. Thus, for some $\omega^{+} \times \omega^{-}$matrix $B(l)$

$$
(\mathscr{R}(\bar{l})-\mathscr{G}(\bar{l})) \Phi_{+}(l)=\chi_{-}(\bar{l}) B^{*}(l) .
$$

Combining the preceding calculations yields

$$
(\mathscr{R}(l)-\mathscr{G}(l)) f(t)=\Phi_{+}(t, l) B(l)\left(f, \chi_{-}(\bar{l})\right)=\left(f, \chi(\bar{l}) B^{*}(l) \Phi_{+}^{*}(t, l)\right) ;
$$

thus,

$$
R(t, \tau, l)-G(t, \tau, l)=\Phi_{+}(t, l) B(l) \chi_{-}^{*}(\tau, \bar{l}) .
$$

Similarly, for some $\omega^{+} \times \omega^{-}$matrix $H(l)$,

$$
R(t, \tau, \bar{l})-G(t, \tau, \bar{l})=\chi_{-}(t, \bar{l}) H^{*}(l) \Phi_{+}^{*}(t, \tau)
$$

and

$$
R^{*}(\tau, t, \bar{l})-G^{*}(\tau, t, \bar{l})=\Phi_{+}(t, l) H(l) \chi_{-}^{*}(\tau, \bar{l}) .
$$

Further, 


$$
\begin{aligned}
B(l) & =\left(\chi_{-}(\bar{l}), \chi_{-}(\bar{l}) B^{*}(l)\right) \\
& =\left(\chi_{-}(\bar{l}),(\mathscr{R}(\bar{l})-\mathscr{G}(\bar{l})) \Phi_{+}(l)\right) \\
& =\left((\mathscr{R}(l)-\mathscr{G}(l)) \chi_{-}(\bar{l}), \Phi_{+}(l)\right) \\
& =\left(\Theta_{+}(l) H(l), \Theta_{+}(l)\right) \\
& =H(l),
\end{aligned}
$$

so that

$$
R^{*}(\tau, t, \bar{l})-G^{*}(\tau, t, \bar{l})=R(t, \tau, l)-G(t, \tau, l) .
$$

Since $\theta_{j}(l) \in \mathscr{E}(l) \subset \mathscr{D}$, for $j=1, \cdots, \omega^{+}$, and $\chi_{k}(l) \in \mathscr{E}(\bar{l}) \subset \mathscr{D}$, for $k$ $=1, \cdots, \omega^{-}$, then $\Theta_{+}(l) B(l) \chi_{-}^{*}(\tau, \bar{l}) \in \mathscr{L}^{2}(a, b)$ for $a<\tau<b, \operatorname{Im} l \neq 0$. Thus $\mathscr{R}(l)$ is an integral operator of Carleman type.

The operator $\mathscr{R}(l)$ will completely satisfy the condition of the theorem when it is shown that $R(t, \tau, l)$ is analytic in $l, \operatorname{Im} l \neq 0$, and $t \neq \tau$. To facilitate the proof of the analyticity of $R(t, \tau, l)$, analytic bases for $\mathscr{E}(l)$ and $\mathscr{E}(\bar{l})$ will be introduced, as in Coddington [5], related to an arbitrary $l_{0}, \operatorname{Im} l_{0}>0$.

Matrices $\Psi_{-}(\bar{l})$ and $\Phi_{+}(l)$ are defined by this process such that the columns of $\Phi_{+}(l)$ form a basis for $\mathscr{E}(l)$ and the columns of $\Psi_{-}(\bar{l})$ form a basis for $\mathscr{E}(\bar{l})$; thus for some nonsingular matrix $T(\bar{l})$,

$$
\Psi_{-}(\bar{l})=\chi_{-}(\bar{l}) T(\bar{l}),
$$

and for some nonsingular matrix $S(l)$

$$
\Phi_{+}(l)=\Theta_{+}(l) S(l) .
$$

Thus,

$$
\begin{aligned}
\Theta_{+}(t, l) B(l) \chi_{-}^{*}(\tau, \bar{l}) & =\Phi_{+}(t, l) S^{-1}(l) B(l)\left(T^{*}(\bar{l})\right)^{-1} \chi_{-}^{*}(\tau, l), \\
& =\Phi_{+}(t, l) C(l) \Psi_{-}(\tau, \bar{l})
\end{aligned}
$$

where $C(l)=S^{-1}(l) B(l)\left(T^{*}(\bar{l})\right)^{-1}$. The matrix $\Phi_{+}(l)$ is analytic in $l$ and $\Psi_{*}^{*}(\bar{l})$ is analytic in $l$ for any compact subset of $\operatorname{Im} l \neq 0$. Thus it remains to show that $C(l)$ satisfies the same conditions of analyticity.

Let $Z$ be an $n \times r$ matrix each of whose columns $z_{k}$ is in $\mathscr{L}^{2}(a, b)$, $k=1, \cdots, r$. Then $\mathscr{R}(l) z_{k}$ is in $\mathscr{D}(l)$ and thus satisfies the boundary condition $(L B)$,

$$
0=\left\langle\left(\mathscr{R}(l) z_{k}\right) V_{*}(l)\right\rangle
$$

the set $\left\{0=\left\langle\left(\mathscr{R}(l) z_{k}\right) V_{*}(l)\right\rangle, k=1, \cdots, r\right\}$ can be written in matrix form as

$$
0=\left\langle(\mathscr{R}(l) Z) V_{*}(l)\right\rangle .
$$

Expanding $\mathscr{R}(l)$, yields 


$$
0=\left\langle(\mathscr{G}(l) Z) V_{*}(l)\right\rangle+\left\langle\left(\Phi_{+}(l) C(l)\left(Z, \Psi_{-}(\bar{l})\right)\right) V_{*}(l)\right\rangle
$$

also

$$
\left\langle\left(\Phi_{+}(l) C(l)\left(Z, \Psi_{-}(\bar{l})\right)\right) V_{*}(l)\right\rangle=\left\langle\Phi_{+}(l) V_{*}(l)\right\rangle C(l)\left(Z, \Psi_{-}(\bar{l})\right) .
$$

Thus,

$$
-\left\langle(\mathscr{G}(l) Z) V_{*}(l)\right\rangle=\left\langle\Phi_{+}(l) V_{*}(l)\right\rangle C(l)\left(Z, \Psi_{-}(\bar{l})\right) .
$$

The matrix $C(l)$ will be analytic if $\left\langle(\mathscr{G}(l) Z) V_{*}(l)\right\rangle$ is analytic, and $\left\langle\Phi_{+}(l) V_{*}(l)\right\rangle$ and $\left(Z, \Psi_{-}(\bar{l})\right)$ are each nonsingular and analytic.

First it can be shown that $\left\langle\Phi_{+}(l) V_{*}(l)\right\rangle$ is nonsingular and analytic. Next, for $\left(Z, \Psi_{-}(\bar{l})\right)$ to be nonsingular $Z$ must be an $n \times \omega^{-}$matrix, it can be verified that for $Z=\Psi_{-}(-i),\left(Z, \Psi_{-}(\bar{l})\right)$ is nonsingular and analytic. Finally, $\left\langle\left(\mathscr{G}(l) \Psi_{-}(-i) V_{*}(l)\right\rangle\right.$ is analytic in $l$ for $\left|l-l_{0}\right|<$ $\operatorname{Im} l_{0} / 2$. Thus

$$
C(l)=-\left\langle\Phi_{+}(l) V_{*}(l)\right\rangle^{-1}\left\langle\left(\mathscr{G}(l) \Psi_{-}(-i) V_{*}(l)\right\rangle\left(\Psi_{-}(-i), \Psi_{-}(l)\right)^{-1}\right.
$$

is analytic and

$$
\Phi_{+}(t, l) C(l) \Phi_{-}^{*}(\tau, \bar{l})=\Theta_{+}(t, l) B(l) \chi_{-}^{*}(\tau, \bar{l})
$$

is analytic in $l$ in a compact subset of $\operatorname{Im} l \neq 0,\left|l-l_{0}\right|<\operatorname{Im} l_{0} / 2$. Theorem 5.2 is now proved, the generalized resolvent $\mathscr{R}(l)$ with kernel $\mathscr{R}(t, \tau, l)$ has been constructed.

\section{The spectral matrix.}

Definition. A matrix $\rho$, (associated with an eigenvalue problem) is a spectral matrix if it satisfies:

(i) $\rho$ is Hermitian,

(ii) $\rho(\Delta)=\rho(\lambda)-\rho(\mu) \geqq 0$ if $\lambda>\mu$, (where $\Delta=[\mu, \lambda]$ ),

(iii) $\rho$ is of bounded variation on every finite $\lambda$ interval.

To develop the spectral matrix associated with the problem $(L-l) u$ $=0$ with the boundary conditions $(L B)$, and thus associated with the generalized resolvent $\mathscr{R}$ and the generalized resolution of the identity $F$, the kernel of $\mathscr{R}(l)$ will be split into two parts,

$$
R(t, \tau, l)=R_{0}(t, \tau, l)+R_{1}(t, \tau, l)
$$

where $R_{0}(t, \tau, l)$ is a certain fundamental matrix for $(L-l) u=0$. Once again, let $\Phi$ be a fundamental matrix for $(L-l) u=0$, satisfying $\Phi(c, l)=I$, for some $c, a<c<b$. Then, as shown in $\S 3$,

$$
[\Phi(t, l) \Phi(t, \bar{l})]=\Phi^{*}(t, \bar{l}) A_{0}(t) \Phi(t, l)=D^{-1},
$$

where $D$ is a nonsingular, constant, skew-Hermitian matrix. Defining 
$R_{0}(t, \tau, l)$ by

$$
R_{0}(t, \tau, l)= \begin{cases}\frac{1}{2} \Phi(t, l) D \Phi^{*}(\tau, \bar{l}), & t \geqq \tau, \\ \frac{1}{2} \Phi(t, l) D^{*} \Phi^{*}(t, \bar{l}), & t_{\star}^{*}<\tau,\end{cases}
$$

then,

$$
R_{0}^{*}(\tau, t, \bar{l})=R_{0}(t, \tau, l)
$$

Also,

$$
R_{0}(t+, t, l)-R_{0}(t-, t, l)=A_{0}^{-1}(t),
$$

which is the same jump that $R(t, \tau, l)$ has at $t=\tau$.

Now, let

$$
R_{1}(t, \tau, l)=R(t, \tau, l)-R_{0}(t, \tau, l) .
$$

Then as a function of $t, R_{1}$ has a continuous first derivative, and $\left(L_{t}-l\right) R_{1}(t, \tau, l)=0$.

From the symmetry property $R_{1}^{*}(\tau, t, \bar{l})=R_{1}(t, \tau, l)$ it follows that for some matrix $\Psi(l)$

$$
R_{1}(t, \tau, l)=\Phi(t, l) \Psi(l) \Phi^{*}(\tau, \bar{l})
$$

THEOREM 6.1. The matrix $\Psi$ is analytic for $\operatorname{Im} l>0, \Psi^{*}(l)=\Psi(\bar{l})$, and $\operatorname{Im} \Psi(l) / \operatorname{Im} l>0$, where $\operatorname{Im} \Psi=\left(\Psi-\Psi^{*}\right) / 2 i$.

Proof. The analyticity of $\Psi$ follows from the choice of $\Phi(c, l)=$ I.

Next,

$$
R_{1}(t, \tau, l)=R_{1}^{*}(\tau, t, \bar{l})
$$

implying

$$
\Phi(t, l) \Psi(l) \Phi^{*}(\tau, \bar{l})=\Phi(t, l) \Psi^{*}(\bar{l}) \Phi^{*}(\tau, \bar{l}),
$$

and, since $\Phi^{-1}$ exists, $\Psi(l)=\Psi^{*}(\bar{l})$, or $\Psi^{*}(l)=\Psi(\bar{l})$.

Let

$$
H(t, \tau, l)=\frac{R(t, \tau, l)-R(t, \tau, \bar{l})}{2 i},
$$

direct computation yields

$$
H(c, c, l)=\operatorname{Im} \Psi(l) .
$$


The proof for $\operatorname{Im} \Psi(l) / \operatorname{Im} l \geqq 0$ now follows as in the proof of Theorem 3 of Coddington [5].

THEOREM 6.2. The matrix $\rho$ defined by

$$
\rho(\lambda)=\lim _{\varepsilon \rightarrow+0} \frac{1}{\pi} \int_{0}^{\lambda} \operatorname{Im} \Psi(\nu+i \varepsilon) d \nu
$$

exists, is nondecreasing and is of bounded variation on any finite interval.

Proof. This follows directly from Theorem 4 of Coddington [5]. The matrix $\rho$ is the spectral matrix associated with the generalized resolvent $\mathscr{R}$ and the generalized resolution of the identity $F$.

7. The generalized resolution of the identity. Let $\rho$ be the spectral matrix derived in $\S 6$, let $\Delta=(\mu, \lambda]$ be a finite interval, and let $F(\Delta)=F(\lambda)-F(\mu)$.

TheOREM 7.1. Let $f \in \mathscr{H}$ and vanish outside a closed bounded subinterval $[c, d]$ of $(a, b)$. If $\mu$ and $\lambda$ are continuity points of $F$, then

$$
F(\Delta) f(t)=\int_{\Delta} \Phi(t, \nu) d \rho(\nu)(f, \Phi(\nu)) .
$$

Proof. It follows from the relationship

$$
(\mathscr{R}(l) f, f)=\int_{-\infty}^{\infty} \frac{d(F(\gamma) f, f)}{\gamma-l}
$$

that

$$
(F(\Delta) f, f)=\lim _{\varepsilon \rightarrow+0} \frac{1}{\pi} \int_{\Delta}(\operatorname{Im} \mathscr{R}(\nu+i \varepsilon) f, f) d \nu
$$

at continuity points $\mu, \lambda$ of $F$. The generalized resolvent $\mathscr{R}(l)$ can be written as $\mathscr{R}(l)=\mathscr{R}_{0}(l)+\mathscr{R}_{1}(l)$, where $\mathscr{R}_{0}(l)$ has kernel $R_{0}(t, \tau, l)$, and $\mathscr{R}_{1}(l)$ has kernel $R_{1}(t, \tau, l)$. Then

$$
\mathscr{R}_{0}(l) f(t)=\int_{a}^{b} R_{0}(t, \tau, l) f(\tau) d \tau=\int_{c}^{d} R_{0}(t, \tau, l) f(\tau) d \tau .
$$

However, $\left(\operatorname{Im} \mathscr{R}_{0}(\nu+i \varepsilon) f, f\right)$ tends to zero $\varepsilon \rightarrow+0$, uniformly in 4. Consequently, it follows that

$$
(F(\Delta) f, f)=\lim _{\varepsilon \rightarrow+0} \frac{1}{\pi} \int_{\Delta}\left(\operatorname{Im} \mathscr{R}_{1}(\nu+i \varepsilon) f, f\right) d \nu,
$$


where

$$
\begin{aligned}
\left(\operatorname{Im} \mathscr{R}_{1}(\nu+i \varepsilon) f, f\right) \\
=(\Phi(\nu), f)\left[\frac{\Psi(\nu+i \varepsilon)-\Psi(\nu-i \varepsilon)}{2 i}\right](f, \Phi(\nu)) \\
\quad+\frac{1}{2 i}\{\Phi(\nu+i \varepsilon), f) \Psi(\nu+i \varepsilon)(f,(\Psi(\nu-i \varepsilon)-\Phi(\nu))) \\
\quad+((\Phi(\nu+i \varepsilon)-\Phi(\nu)), f) \Psi(\nu+i \varepsilon)(f, \Psi(\nu))\} \\
\quad+\frac{1}{2 i}\{(\Phi(\nu), f) \Psi(\nu-i \varepsilon)(f,(\Phi(\nu)-\Phi(\nu+i \varepsilon))) \\
\quad+((\Phi(\nu)-\Phi(\nu-i \varepsilon)), f) \Psi(\nu-i \varepsilon)(f, \Phi(\nu+i \varepsilon))\} \\
=T_{1}+T_{2}+T_{3},
\end{aligned}
$$

where $T_{i}=T_{i}(\nu, \varepsilon, f)$.

In Lemma 3 of Straus [13], it is shown that

$$
\lim _{\varepsilon \rightarrow+0} \frac{1}{\pi} \int_{4} T_{2}(\nu) d \nu=\lim _{\varepsilon \rightarrow+0} \frac{1}{\pi} \int_{4} T_{3}(\nu) d \nu=0 .
$$

Finally, for $T_{1}$,

$$
\begin{gathered}
\lim _{\varepsilon \rightarrow 0} \frac{1}{\pi} \int_{\Delta}(\Phi(\nu), f) \operatorname{Im} \Psi(\nu+i \varepsilon)(f, \Phi(\nu)) d \nu \\
\quad=\int_{\Delta}(\Phi(\nu), f) d \rho(\nu)(f, \Phi(\nu)) \\
\quad=\int_{\Delta}(f, \Phi(\nu)) * d \rho(\nu)(f, \Phi(\nu)),
\end{gathered}
$$

and therefore,

$$
(F(\Delta) f, f)=\int_{a}^{b} f^{*}(t)\left[\int_{\Delta} \Phi(t, \nu) d \rho(\nu)(f, \Phi(\nu))\right] d t .
$$

Since this representation must hold for all $f \in \mathscr{H}$ which vanish outside closed finite subintervals of $(a, b)$,

$$
\left.F(\Delta) f(t)=\int_{\Delta} \Phi(t, \nu) d \rho(\nu) f, \Phi(\nu)\right)
$$

for all such $f$.

Thus the generalized resolutions of the identity associated with the first order system of differential operators $L x(t)=A_{0}(t) x^{\prime}(t)+$ $A(t) x(t)$ can be represented explicitly in terms of a certain fundamental matrix $\Phi$ and an associated spectral matrix $\rho$.

8. The expansion and completeness relations. Expansion and completeness relations can be defined in terms of the spectral matrix 
$\rho$ and the fundamental matrix $\Phi$. For two vectors $\hat{\alpha}, \widehat{\beta}$, an inner product is defined in terms of $\rho$ by

$$
(\widehat{\alpha}, \widehat{\beta})_{\rho}=\int_{-\infty}^{\infty} \widehat{\beta}^{*}(\nu) d \rho(\nu) \widehat{\alpha}(\nu) .
$$

Thus a norm can be defined by

$$
\|\hat{\alpha}\|_{\rho}=(\widehat{\alpha}, \hat{\alpha})_{\rho}^{1 / 2} .
$$

The Hilbert space $\mathscr{L}^{2}(\rho)$ is defined by

$$
\mathscr{L}^{2}(\rho)=\left\{\hat{\alpha}:\|\hat{\alpha}\|_{\rho}<\infty\right\} .
$$

Defining a mapping from $\mathscr{L}^{2}(a, b)$ into $\mathscr{L}^{2}(\rho)$ by

$$
\hat{f}(\nu)=(f, \Phi(\nu))=\int_{a}^{b} \Phi^{*}(t, \nu) f(t) d t,
$$

the expansion and completeness relations have the following form:

$$
f(t)=\left(\hat{f}, \Phi^{*}(t)\right)_{\rho}=\int_{-\infty}^{\infty} \Phi(t, \nu) d \rho(\nu) \hat{f}(\nu) \quad \text { (expansion) }
$$

and

$$
\|f\|=\|\hat{f}\|_{\rho} \quad \text { (completeness) }
$$

\section{REFERENCES}

1. N. I. Akhiezer and I. M. Glazman, Theory of Linear Operators in Hilbert Space (English Translation) F. Ungar Publishing Co., N. Y., Vol. 1 and Vol. 2, 1961, 1963.

2. F. Brauer, Spectral theory for linear systems of differential equations, Pacific J. Math., V. 10 (1960), 17-34.

3. E. A. Coddington, The spectral representation of ordinary self-adjoint differential operators, Annals of Math., 60 (July 1954), 192-211.

4. , The spectral matrix and Green's function for singular self-adjoint boundary value problems, Canad. Math., 6 (1954), 169-185.

5. , Generalized resolutions of the identity for symmetric ordinary differential operators, Annals of Math., 68 (Sept. 1958) 378-392.

6. L and N. Levinson, Theory of Ordinary Differential Equations, McGrawHill Book Co., Inc., 1955.

7. M. A. Naimark, Spectral functions of a symmetric operator, Izvest. Akad. Nauk. S. S. S. R. Ser. Mat., 4 (1940), 277-318.

8. F. Riesz and B. Sz-Nagy, Functional Analysis, (English Translation) F. Ungar Publishing Co., N. Y., 1955.

9. M. H. Stone, Linear Transformations in Hilbert Space, Amer. Math. Soc. Colloqu. Publ., 1932.

10. A. V. Straus, On generalized resolvents of a symmetric operator, Dok. Akad. Nauk. S. S. S. R., 71 (1950), 241-244.

11 . , On the theory of generalized resolvents of a symmetric operator, Dok. Akad. Nauk. S. S. S. R., 78 (1951), 217-220. 
12. Generalized resolvents of symmetric operators, Izvest. Akad. Nauk. S. S. S. R., Ser. Mat., 18 (1954), 51-86.

13. On spectral functions of differential operators, Izvest. Akad. Nauk S. S. S. R., Ser. Mat., 19 (1955), 201-220.

Received September 4, 1970 and in revised form June 19, 1971.

Pacific Missile Range, Point Magu, California 


\section{PACIFIC JOURNAL OF MATHEMATICS}

\section{EDITORS}

H. SAMELSON

Stanford University

Stanford, California 94305

C. R. HobBY

University of Washington

Seattle, Washington 98105
J. DugundjI

Department of Mathematics

University of Southern California

Los Angeles, California 90007

RICHARD ARENS

University of California

Los Angeles, California 90024

\section{ASSOCIATE EDITORS}

E. F. BECKENBACH

B. H. NeUMaNN

F. WOLF

K. YoshidA

\section{SUPPORTING INSTITUTIONS}

UNIVERSITY OF BRITISH COLUMBIA

CALIFORNIA INSTITUTE OF TECHNOLOGY

UNIVERSITY OF CALIFORNIA

MONTANA STATE UNIVERSITY

UNIVERSITY OF NEVADA

NEW MEXICO STATE UNIVERSITY

OREGON STATE UNIVERSITY

UNIVERSITY OF OREGON

OSAKA UNIVERSITY
UNIVERSITY OF SOUTHERN CALIFORNIA STANFORD UNIVERSITY

UNIVERSITY OF TOKYO

UNIVERSITY OF UTAH

WASHINGTON STATE UNIVERSITY UNIVERSITY OF WASHINGTON

$\stackrel{*}{*} \stackrel{*}{*} \stackrel{*}{*}$ AMERICAN MATHEMATICAL SOCIETY
NAVAL WEAPONS CENTER 


\section{Pacific Journal of Mathematics}

Vol. 39 , No. 1

May, 1971

Charles A. Akemann, A Gelfand representation theory for $C^{*}$-algebras ....

Sorrell Berman, Spectral theory for a first-order symmetric system of

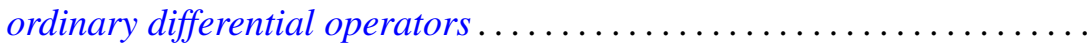

Robert L. Bernhardt, III, On splitting in hereditary torsion theories ........

J. L. Brenner, Geršgorin theorems, regularity theorems, and bounds for determinants of partitioned matrices. II. Some determinantal identities ..........................................

Robert Morgan Brooks, On representing $F^{*}$-algebras .............. 51

Lawrence Gerald Brown, Extensions of topological groups........... 71

Arnold Barry Calica, Reversible homeomorphisms of the real line ........ 79

J. T. Chambers and Shinnosuke Oharu, Semi-groups of local Lipschitzians in

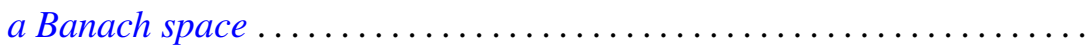

Thomas J. Cheatham, Finite dimensional torsion free rings .............

Byron C. Drachman and David Paul Kraines, A duality between

transpotence elements and Massey products ...................

Richard D. Duncan, Integral representation of excessive functions of a

Markov process ......................................

George A. Elliott, An extension of some results of Takesaki in the reduction

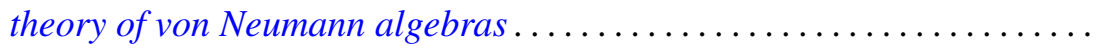

Peter C. Fishburn and Joel Spencer, Directed graphs as unions of partial

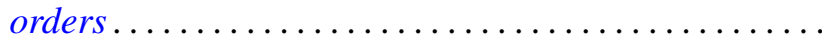

Howard Edwin Gorman, Zero divisors in differential rings ...

Maurice Heins, A note on the Löwner differential equations...

Louis Melvin Herman, Semi-orthogonality in Rickart rings. .

David Jacobson and Kenneth S. Williams, On the solution of linear G.C.D.

equations

Michael Joseph Kallaher, On rank 3 projective planes ... . .

Donald Paul Minassian, On solvable $O^{*}$-groups ...........

Nils Øvrelid, Generators of the maximal ideals of $A(\bar{D})$

Mohan S. Putcha and Julian Weissglass, A semilattice decomposition into

semigroups having at most one idempotent ............

Robert Raphael, Rings of quotients and $\pi$-regularity ....

J. A. Siddiqi, Infinite matrices summing every almost periodic sequence. .

Raymond Earl Smithson, Uniform convergence for multifunctions ...

Thomas Paul Whaley, Mulitplicity type and congruence relations in

universal algebras...

Roger Allen Wiegand, Globalization theorems for locally finitely generated modules... 